\title{
Clinical utility gene card for: Smith-Lemli-Opitz Syndrome [SLOS]
}

\author{
Martina Witsch-Baumgartner ${ }^{\star, 1}$, Hilary Sawyer ${ }^{2}$ and Dorothea Haas ${ }^{3}$ \\ European Journal of Human Genetics (2013) 21, doi:10.1038/ejhg.2012.255; published online 16 January 2013
}

\section{DISEASE CHARACTERISTICS}

1.1 Name of the disease (synonyms)

Smith-Lemli-Opitz Syndrome.

SLOS.

SLO Syndrome.

RSH Syndrome.

Rutledge Lethal Multiple Congenital Anomaly Syndrome.

Polydactyly, Sex Reversal, Renal Hypoplasia, and Unilobar Lung.

Lethal Acrodysgenital Syndrome.

\subsection{OMIM\# of the disease}

270400.

1.3 Name of the analysed genes or DNA/chromosome segments DHCR7.

1.4 OMIM\# of the gene(s)

602858 .

\subsection{Mutational spectrum}

The Smith-Lemli-Opitz syndrome, an autosomal recessive metabolic malformation/mental retardation syndrome is caused by mutations in the DHCR7 gene, which encodes the $3 \beta$-hydroxysteroid- $\Delta 7$-reductase (DHCR7, E.C.1.3.1.21). ${ }^{1}$ More than one-hundred SLOS causal mutations have been described so far (database in HGVS: http:// lovd.i-med.ac.at/home.php, HGMD: www.hgmd.org/). The estimated frequency of the disease in European populations is 1:10000$1: 40000 .^{2}$ The syndrome is characterized by facial dysmorphism, palatal clefting, 2,3-toe syndactyly, postaxial polydactyly, malformations of heart, kidney, genitalia, and lungs; occasional holoprosencephaly and other CNS malformations, as well as severe to profound mental retardation, and failure to thrive (Smith et al. ${ }^{3}$ ). Later in life, most patients have behavioural abnormalities such as aggressive and self-injurious behaviour and autism like characteristics.

Characterisation of the mutational spectrum of the DHCR7 gene was possible after studying $>100$ SLOS patients (Witsch-Baumgartner $\mathrm{M}$ et $a l^{4,5}$ Correa-Cerro et $a l^{6}$ ). Until now $>100$ different mutations: nonsense (eg, p.Trp151*), deletions (eg, c.720-735del and c.385-412IVS5 + 1-5del, HGVS: c.385_412+5del), splice site mutations (eg, c.964-1G $>$ C) and missense mutations have been described. The most frequent class of mutations are missense mutations. Approximately half of them involve conserved amino acids. They are located in or near the transmembrane domains, in the fourth cytoplasmic loop or in the C terminal region of the DHCR7 protein. ${ }^{4}$ More than $95 \%$ SLOS patients are homozygous or compound heterozygous for DHCR7 point mutations.

A correlation of the genotype with the SLOS phenotype ${ }^{7}$ demonstrates that patients carrying homozygous or compound heterozygous functional null DHCR7 alleles have the most severe phenotypes. Missense mutations may be associated with residual activity and hence milder phenotypes. Unfortunately even with a degree of genotype-phenotype correlation, it is not possible to predict the phenotype by knowing the genotype and vice versa. Other factors appear to influence the phenotype including maternal apolipoprotein E genotype (Witsch-Baumgartner $\mathrm{M}$ et $a l^{8}$ ).

\subsection{Analytical methods}

Detection of homozygous/heterozygous mutations in the DHCR7 gene by amplification of coding exon sequences (exons 3-9) with bordering intron sequences by PCR of genomic DNA and complete sequencing of these fragments.

Depending on ethnic background, it might be reasonable to start analysis specifically for frequent mutations for instance in British patients for the frequent mutation c.964-1G $>$ C or in Polish patients for the p.Trp $151^{\star}$ mutation. This might be done by mutation-specific PCR methods or by sequencing the corresponding exon.

Targeted sequence analysis referrals for carrier testing or prenatal diagnosis for familial mutations.

\subsection{Analytical validation}

Obtained PCR fragments have been analyzed in $\sim 50$ DNA samples by sequencing. All variants had been tested for frequency in 50-100 DNA samples of corresponding population background. If possible, in each case with homozygosity or compound heterozygosity for mutations they are tested in parental DNAs.

External quality assurance (EQA) should be carried out for DNA sequencing (URL: http://www.emqn.org/).

\subsection{Estimated frequency of the disease}

(Incidence at birth ('birth prevalence') or population prevalence) The reported birth prevalence varies depending on geographic region. In middle Europe, it is approx. 1:20000-1:60000 (Kelley and

\footnotetext{
${ }^{1}$ Department of Medical Genetics, Molecular and Clinical Pharmacology, Institute of Human Genetics, Medical University Innsbruck, Innsbruck, Austria; ${ }^{2}$ Bristol Genetics Laboratory, North Bristol NHS Trust, Bristol, UK; ${ }^{3}$ Zentrum für Kinder- und Jugendmedizin, Klinik I, Sektion für angeborene Stoffwechselerkrankungen, Universitätsklinikum Heidelberg, Heidelberg, Germany

*Correspondence: Dr M Witsch-Baumgartner, Institute of Human Genetics, Medical University Innsbruck, Schoepfstrasse 41, Innsbruck A-6020, Austria. Tel: + 43-51-70545; Fax: +43-512-73510; E-mail: witsch-baumgartner@i-med.ac.at
} 
Hennekam $2000^{9}$ and Ryan et al. $1998^{10}$ ). In the US, it is 1:60000. In Eastern European population, prevalence might be as high as 1:16.000. In African or Asian populations, SLOS is nearly unknown. The spectrum of phenotype in Smith-Lemli-Opitz syndrome is wide and cases may be underdiagnosed. Clinical incidence does not appear to be as high as predicted from carrier frequency possibly because of underascertainment of mild cases, prenatal loss, or neonatal mortality before diagnosis.

\subsection{If applicable, prevalence in the ethnic group of investigated} person

Birth prevalence is significantly increased in populations of Eastern European origin: 1:16000.

Birth prevalence is significantly low in African and Asian populations.

\subsection{Diagnostic setting}

A. (Differential) diagnostics

Yes

B. Predictive testing

C. Risk assessment in relatives

D. Prenatal

Comment:

Predictive diagnostics are not offered because the disease already manifests at birth. DHCR7 mutation analysis for risk assessment in possible carriers of the disease is important as biochemical carrier testing is challenging and specialized.

\section{TEST CHARACTERISTICS}

\begin{tabular}{|c|c|c|c|c|}
\hline & \multicolumn{2}{|c|}{ Genotype or disease } & \multirow{2}{*}{$\begin{array}{l}\text { A: True positives } \\
\text { B: False positives }\end{array}$} & \multirow{2}{*}{$\begin{array}{l}\text { C: False negative } \\
\text { D: True negative }\end{array}$} \\
\hline & Present & Absent & & \\
\hline \multicolumn{5}{|l|}{ Test } \\
\hline Positive & $A$ & B & $\begin{array}{l}\text { Sensitivity: } \\
\text { Specificity: }\end{array}$ & $\begin{array}{l}A /(A+C) \\
D /(D+B)\end{array}$ \\
\hline Negative & C & $D$ & $\begin{array}{l}\text { Positive predictive value: } \\
\text { Negative predictive value: }\end{array}$ & $\begin{array}{l}A /(A+B) \\
D /(C+D)\end{array}$ \\
\hline
\end{tabular}

\subsection{Analytical sensitivity}

(proportion of positive tests if the genotype is present)

Depending on the method used almost $100 \%$ for DHCR7 mutations.

\subsection{Analytical specificity}

(proportion of negative tests if the genotype is not present)

Depending on the method used almost $100 \%$.

\subsection{Clinical sensitivity}

(proportion of positive tests if the disease is present)

The clinical sensitivity can be dependent on variable factors such as age or family history. In such cases, a general statement should be given, even if quantification can only be made case by case.

$>95 \%$.

SLOS patients have an increased serum concentration of 7dehydrocholesterol (7-DHC) and 8-dehydrocholesterol (8-DHC), which is pathognomonic of the disease. If raised 7- and 8-DHC levels are present indicating SLOS, the clinical sensitivity is nearly $100 \%$.

\subsection{Clinical specificity}

(proportion of negative tests if the disease is not present)

The clinical specificity can be dependent on variable factors such as age or family history. In such cases a general statement should be given, even if quantification can only be made case by case.

$>99.9 \%$.

If 7- and 8-DHC levels determined by GC-MS analysis are within the normal range, the clinical specificity is nearly $100 \%$. The plasma cholesterol concentration is decreased in most SLOS patients; however, $\sim 10 \%$ of patients have normal cholesterol levels (Nowaczyk and Waye 2001 11 , Kelley and Hennekam 2000 ${ }^{9}$ ). Therefore routine measurement of cholesterol alone is not a suitable screening method.

\subsection{Positive clinical predictive value}

(life time risk to develop the disease if the test is positive)

Estimated $>99 \%$ with homozygosity or compound heterozygosity for DHCR7 mutations.

\subsection{Negative clinical predictive value} (probability not to develop the disease if the test is negative) Assume an increased risk on the basis of family history for a nonaffected person. Allelic and locus heterogeneity may need to be considered.

Index case in that family had been tested: $>99 \%$ with homozygosity or compound heterozygosity for DHCR7 mutations detected in the index case.

Index case in that family had not been tested:

$$
>99 \% \text {. }
$$

\section{CLINICAL UTILITY}

3.1 (Differential) diagnostics: The tested person is clinically affected

(To be answered if in 1.10 'A' was marked)

\subsubsection{Can a diagnosis be made other than through a genetic test?}

$\begin{array}{ll}\text { No } \\ \text { Yes } \mathbb{\text { (continue with 3.1.4) }} \\ \text { Clinically } & \square \\ \text { Imaging } & \square \\ \text { Endoscopy } & \square \\ \text { Biochemistry } & \square \\ \text { Electrophysiology } & \square \\ \text { Other (please describe) } & \text { Yes, by quantification of 7- and 8-DHC in } \\ & \text { addition to cholesterol by GC-MS }\end{array}$

\subsubsection{Describe the burden of alternative diagnostic methods to the} patient

Mildly affected patients may have 7-DHC concentrations in the upper normal range and only marginally elevated 8-DHC concentrations (Langius et al. 2003 ${ }^{12}$ ). Very rarely, even patients with a classical phenotype have borderline sterol concentrations. ${ }^{13}$ Therefore genetic testing should be done in all patients with elevated concentrations of 7- or 8-DHC, even if the elevation is only marginal. 
3.1.3 How is the cost effectiveness of alternative diagnostic methods to be judged?

Genetic testing is significantly more expensive than GC-MS analysis. Therefore determination of sterols by GC-MS should be used as the primary screening method. In any case, genetic testing should be used whenever the diagnosis is problematic. In future in view of the decreasing cost of DNA analysis and of the more powerful diagnostic capacity of mutation analysis in SLOS genetic testing will be favored.

3.1.4 Will disease management be influenced by the result of a genetic test?

\begin{tabular}{ll}
\hline No $\square$ & \\
Therapy & \\
& Dietary cholesterol supplementation results in improved \\
& growth and behavior in most patients. Photosensitivity \\
& and polyneuropathy improve significantly (Azurdia \\
& et al, ${ }^{14}$ Starck et al. 199915$)$. Unfortunately, there is no \\
& effect of cholesterol supplementation on intrinsic \\
& cognitive abilities. Mildly affected patients may benefit \\
& from treatment with statins, whereas severely affected \\
& patients are prone to serious side effects. However, the \\
& differentiation between severely and mildly affected \\
& patients can be reliably determined by biochemical \\
analysis (ratio of (7 +8 -DHC)/cholesterol at time of \\
diagnosis). ${ }^{16}$ \\
Life expectancy in SLOS is primarily determined by the \\
degree of prenatally acquired internal malformations, \\
which are most severe in patients with homozygosity or \\
compound heterozygosity for two functional null mutations. \\
Those patients usually die in the neonatal period. The \\
individual course in patients with other mutations can be \\
better related to the published experience and differentiated \\
from other intellectual disabilities. \\
The result of genetic tests will influence the counseling \\
of parents. If two functional null mutations are present, \\
invasive interventions such as surgical correction of severe \\
heart defects or liver transplantation in severe hepatopathy \\
will not improve overall survival.
\end{tabular}

3.2 Predictive setting: The tested person is clinically unaffected but carries an increased risk on the basis of family history

(To be answered if in 1.10 'B' was marked)

\author{
3.2.1 Will the result of a genetic test influence lifestyle and \\ prevention? \\ If the test result is positive (please describe) \\ If the test result is negative (please describe)
}

3.2.2 Which options in view of lifestyle and prevention does a person at-risk have if no genetic test has been done (please describe)? Not applicable.

3.3 Genetic risk assessment in family members of a diseased person (To be answered if in 1.10 'C' was marked)

\subsubsection{Does the result of a genetic test resolve the genetic situation in that family?}

Yes, it confirms the genetic transmission and is prerequisite for genetic counseling of family members.
3.3.2 Can a genetic test in the index patient save genetic or other tests in family members?

No more tests are required in the patient to secure his diagnosis of SLOS unless sterol levels are equivocal. However, except for the parents, the risk of relatives is uncertain without individual genetic tests.

3.3.3 Does a positive genetic test result in the index patient enable a predictive test in a family member?

Yes (in most cases, however, a predictive test is only performed for diagnosing or excluding heterozygosity or prenatal analysis).

\subsection{Prenatal diagnosis}

(To be answered if in 1.10 ' $\mathrm{D}$ ' was marked)

\subsubsection{Does a positive genetic test result in the index patient enable a prenatal diagnosis?}

Yes.

\section{IF APPLICABLE, FURTHER CONSEQUENCES OF TESTING}

Please assume that the result of a genetic test has no immediate medical consequences. Is there any evidence that a genetic test is nevertheless useful for the patient or his/her relatives? (Please describe)

Genetic diagnosis renders continuous diagnostics (clinical and others) unnecessary and confirms the mode of inheritance in a clinically and genetically heterogeneous group of intellectual disability with malformations and growth retardation. Heterozygote tests in relatives, prognostic statements in patients and prenatal diagnosis in pregnancies at risk become possible as a consequence. ${ }^{17,18}$

\section{CONFLICT OF INTEREST}

The authors declare no conflict of interest.

\section{ACKNOWLEDGEMENTS}

This work was supported by EuroGentest2 (Unit 2: 'Genetic testing as part of health care'), a Coordination Action under FP7 (Grant Agreement Number 261469) and the European Society of Human Genetics.

1 Tint GS, Irons M, Elias ER et al: Defective cholesterol biosynthesis associated with the Smith-Lemli-Opitz syndrome. N Engl J Med 1994; 330: 107-113.

2 Witsch-Baumgartner M, Ciara E, Löffler J et al: Frequency gradients of DHCR7 mutations in patients with Smith-Lemli-Opitz syndrome in Europe: evidence for different origins of common mutations. Eur J Hum Genet 2001a; 9: 45-50.

3 Smith DW, Lemli L, Opitz JM: A newly recognized syndrome of multiple congenital anomalies. J Pediatr 1964; 64: 210-217.

4 Witsch-Baumgartner M, Loeffler J, Utermann G: Mutations in the human DHCR7 gene. Hum Mutat 2001b; 17: 172-182.

5 Witsch-Baumgartner $\mathrm{M}$, Clayton $\mathrm{P}$, Clusellas $\mathrm{N}$ et al: Identification of 14 novel mutations in DHCR7 causing the Smith-Lemli-Opitz syndrome and delineation of the DHCR7 mutational spectra in Spain and Italy. Hum Mutat 2005; 25: 412.

6 Correa-Cerro LS, Porter FD: 3beta-hydroxysterol Delta7-reductase and the SmithLemli-Opitz syndrome. Mol Genet Metab 2005; 84: 112-126.

7 Witsch-Baumgartner M, Fitzky BU, Ogorelkova $\mathrm{M}$ et al: Mutational spectrum in the Delta7-sterol reductase gene and genotype-phenotype correlation in 84 patients with Smith-Lemli-Opitz syndrome. Am J Hum Genet 2000; 66: 402-412.

8 Witsch-Baumgartner M, Gruber M, Kraft HG et al: Maternal ApoE genotype is a modifier of the Smith-Lemli-Opitz syndrome. J Med Genet 2004; 42: 350-357.

9 Kelley RI, Hennekam RC: The Smith-Lemli-Opitz syndrome. J Med Genet 2000; 37: 321-335.

10 Ryan AK, Bartlett K, Clayton P et al: Smith-Lemli-Opitz syndrome: a variable clinical and biochemical phenotype. J Med Genet 1998; 35: 558-565.

11 Nowaczyk MJ, Waye JS: The Smith-Lemli-Opitz syndrome: a novel metabolic way of understanding developmental biology, embryogenesis, and dysmorphology. Clin Genet 2001; 59: 375-386.

12 Langius FA, Waterham HR, Romeijn GJ et al: Identification of three patients with a very mild form of Smith-Lemli-Opitz syndrome. Am J Med Genet A 2003; 122A: 24-29. 
13 Haas D, Armbrust S, Haas JP et al: Smith-Lemli-Opitz syndrome with a classical phenotype, oesophageal achalasia and borderline plasma sterol concentrations. J Inherit Metab Dis 2005; 28: 1191-1196.

14 Azurdia RM, Anstey AV, Rhodes LE: Cholesterol supplementation objectively reduces photosensitivity in the Smith-Lemli-Opitz syndrome. Br J Dermatol 2001; 144: 143-145.

15 Starck L, Bjorkhem I, Ritzen EM, Nilsson BY, von Dobeln U: Beneficial effects of dietary supplementation in a disorder with defective synthesis of cholesterol. A case report of a girl with Smith-Lemli-Opitz syndrome, polyneuropathy and precocious puberty. Acta Paediatr 1999; 88: 729-733.
16 Haas D, Garbade SF, Vohwinkel C et al: Effects of cholesterol and simvastatin treatment in patients with Smith-Lemli-Opitz syndrome (SLOS). J Inherit Metab Dis 2007; 30: 375-387.

17 Gaoua W, Wolf C, Chevy F, Ilien F, Roux C: Cholesterol deficit but not accumulation of aberrant sterols is the major cause of the teratogenic activity in the Smith-Lemli-Opitz syndrome animal model.. J Lipid Res 2000; 41: 637-646.

18 Kelley RI: Diagnosis of Smith-Lemli-Opitz syndrome by gas chromatography/mass spectrometry of 7-dehydrocholesterol in plasma, amniotic fluid and cultured skin fibroblasts. Clin Chim Acta 1995; 236: 45-58. 